\title{
Impact of a Population Health Management Intervention on Disparities in Cardiovascular Disease Control
}

\author{
Aisha James, $M D^{7}$, Seth A. Berkowitz, MD MPH',3, Jeffrey M. Ashburner, $P h D, M P H^{2,3}$, \\ Yuchiao Chang, $P h D^{2,3}$, Daniel M. Horn, MD',3,4, Sandra M. O'Keefe, $\mathrm{MPH}^{4}$, \\ and Steven J. Atlas, MD, MPH ${ }^{2,3}$
}

${ }^{1}$ Harvard/Massachusetts General Hospital Medicine-Pediatrics Residency Program, Boston, MA, USA; 2 Division of General Internal Medicine, Massachusetts General Hospital, Boston, MA, USA; ${ }^{3}$ Harvard Medical School, Boston, MA, USA; ${ }^{4}$ Massachusetts General Physicians Organization, Massachusetts General Hospital, Boston, MA, USA.

BACKGROUND: Healthcare systems use population health management programs to improve the quality of cardiovascular disease care. Adding a dedicated population health coordinator (PHC) who identifies and reaches out to patients not meeting cardiovascular care goals to these programs may help reduce disparities in cardiovascular care.

OBJECTIVE: To determine whether a program that used PHCs decreased racial/ethnic disparities in LDL cholesterol and blood pressure (BP) control.

DESIGN: Retrospective difference-in-difference analysis. PARTICIPANTS: Twelve thousdand five hundred fifty-five primary care patients with cardiovascular disease (cohort for LDL analysis) and 41,183 with hypertension (cohort for BP analysis).

INTERVENTION: From July 1, 2014-December 31, 2014, 18 practices used an information technology (IT) system to identify patients not meeting LDL and BP goals; 8 practices also received a PHC. We examined whether having the PHC plus IT system, compared with having the IT system alone, decreased racial/ethnic disparities, using difference-in-difference analysis of data collected before and after program implementation.

MAIN MEASURES: Meeting guideline concordant LDL and BP goals.

KEY RESULTS: At baseline, there were racial/ethnic disparities in meeting LDL $(p=0.007)$ and BP $(p=0.0003)$ goals. Comparing practices with and without a PHC, and accounting for pre-intervention LDL control, nonHispanic white patients in PHC practices had improved odds of LDL control (OR 1.20 95\% CI 1.09-1.32) compared with those in non-PHC practices. Non-Hispanic black (OR 1.15 95\% CI 0.80-1.65) and Hispanic (OR 1.29 95\% CI 0.66-2.53) patients saw similar, but nonsignificant, improvements in LDL control. For BP control, non-Hispanic white patients in PHC practices (versus non-PHC) improved (OR 1.13 95\% CI 1.05-1.22). Non-

Electronic supplementary material The online version of this article (https://doi.org/10.1007/s11606-017-4227-3) contains supplementary material, which is available to authorized users.

Received June 20, 2017

Revised September 7, 2017

Accepted October 26, 2017

Published online January 8, 2018
Hispanic black patients (OR 1.17 95\% CI 0.94-1.45) saw similar, but non-statistically significant, improvements in BP control, but Hispanic (OR 0.90 95\% CI 0.59-1.36) patients did not. Interaction testing confirmed that disparities did not decrease $(p=0.73$ for LDL and $p=0.69$ for $\mathrm{BP})$.

CONCLUSIONS: The population health management intervention did not decrease disparities. Further efforts should explicitly target improving both healthcare equity and quality.

Clinical Trials \#: NCT02812303 (ClinicalTrials.gov).

KEY WORDS: population health management; racial and ethnic disparities in care; hypertension; hyperlipidemia; cardiovascular disease. $\mathrm{J}$ Gen Intern Med 33(4):463-70 DOI: $10.1007 /$ s11606-017-4227-3

(c) Society of General Internal Medicine 2018

ardiovascular disease is the leading cause of death in men $\mathcal{C}$ and women of all races, ethnicities, and socioeconomic classes in the USA, ${ }^{1}$ but the burden of cardiovascular disease is greater among racial/ethnic minority groups, with $17 \%$ of non-Hispanic black and $13 \%$ of Hispanic adults having poor cardiovascular health as compared to $11 \%$ of non-Hispanic white adults. ${ }^{2}$ Hypertension and dyslipidemia are key risk factors for cardiovascular disease, and improving control of these risk factors plays an important role in both primary and secondary prevention of cardiovascular disease. ${ }^{3}$ However, the Centers for Disease Control and Prevention report that only $48 \%$ of patients with hypertension and $29.5 \%$ of patients with dyslipidemia had their conditions controlled. ${ }^{4,5}$ Rates of control are often worse among patients who identify as a racial or ethnic minority. ${ }^{4-6}$ For example, in 2010, rates of blood pressure control were substantially different among nonHispanic white adults $(56.3 \%)$ compared with non-Hispanic black $(47.9 \%)$ and Hispanic adults with hypertension $(40.7 \%) .^{6}$ In this light, reducing racial/ethnic disparities in blood pressure and cholesterol control, along with improving blood pressure and cholesterol control overall, is a national public health priority. ${ }^{7}$ 
Population health management includes programs that seek to identify and reach out to patients who are not meeting care goals. They are increasingly being used to improve the quality of chronic disease prevention and management across large healthcare delivery systems. ${ }^{8-10}$ These programs vary by institution, but often include a population health information technology system, which helps identify patients not at goal using clinical record data and facilitates outreach to these patients. Additional components may include educational materials and a dedicated population health management workforce, sometimes called population health coordinators, that interfaces with both providers and patients, relieving other clinical staff from some of the population health management work. Supporters have noted that these population health management programs have the potential to reduce disparities through several mechanisms. ${ }^{11-19}$ Provider reminders, audit and feedback, and decision support tools could help raise awareness of existing disparities and limit biased decisionmaking. Support staff, including dedicated personnel, could help to reach out to patients and overcome barriers to care, which may be more common in vulnerable groups, and provide further education. However, if such programs do not directly address disparities, they may allow disparities to persist or even worsen.

To help understand these issues better, we sought to examine the impact of implementing a multifaceted population health intervention on racial/ethnic disparities in blood pressure and cholesterol control. This program used several strategies that had been highlighted in a roadmap to reduce racial/ ethnic disparities, including providing reminders and feedback, restructuring the care team, and use of technology. ${ }^{20}$ Therefore, we hypothesized that such a program would decrease racial/ethnic disparities in blood pressure and cholesterol control.

\section{METHODS}

\section{Study Setting and Sample}

The intervention has been previously described in detail. ${ }^{10}$ In brief, the study was conducted in a network of 18 primary care practices that used the same population health management information technology system (TopCare, SRG Technology). This system identifies what quality measures a patient is eligible for, whether a patient is meeting their goals, and facilitates outreach to the patient. As previously described, included patients were attributed to a practice using a validated algorithm if they had a visit in the 3 years prior to the start of the study (July 1, 2014) or during the 6 month study period (ending December 31, 2014). ${ }^{10}$ Patients included in the low-density lipoprotein (LDL) cholesterol analyses had cardiovascular disease as determined by a previously validated algorithm ${ }^{21,22}$ that used diagnosis codes and problem list items from the patient's electronic health record. Patients included in the blood pressure (BP) analyses had hypertension as assessed by a previously validated algorithm ${ }^{21}$ using similar data elements. Participants could be included in both analyses if they met criteria for both conditions. Demographic data were obtained from the electronic health record. Participants could self-identify into the following racial/ethnic categories: non-Hispanic white, non-Hispanic black, Hispanic, Asian, Other, or Multi-racial. Due to the small sample size in Asian, Other, and Multi-racial groups, we limited the analysis to non-Hispanic white, non-Hispanic black, and Hispanic participants.

The institutional review board at Partners Healthcare approved this project's use of secondary data collected in routine clinical care.

\section{Intervention}

TopCare is a population health management information technology system that has been in use since 2011 in the primary care practices for cancer screening. In conjunction with TopCare, a pilot program began on July 1, 2014, and continued through December 31, 2014. There were two versions of the program. In version one, the intervention group, the population health management information technology system was expanded from being used for cancer screening goals to also being used for cardiovascular care goals (described below). In addition, version one included the assignment of central population health coordinators (PHCs) to individual practices. The PHC proactively identified patients not at goal, without the need for a clinician to order this service. The PHC helped handle administrative issues for patients who were not meeting disease control metrics, such as outreach for appointment scheduling, ordering overdue laboratory testing, chart reviews, and obtaining home blood pressure values and outside tests/laboratories. The PHCs regularly met ("huddled") with physicians to review those patients who required clinical intervention and to develop an action plan. The PHC worked within the organization's commitment to culturally competent care and provided culturally and linguistically appropriate services, including the use of certified medical interpreters when needed. The PHCs were not trained patient navigators or community health workers. There were four PHCs to cover eight clinical sites. In version two of the program, the nonintervention practices without a population health coordinator (non-PHC), the population health management information technology system was still used, but there were no dedicated personnel assigned - the tasks of helping patients meet quality of care goals were handled by usual clinic staff and medical providers in addition to their regular duties.

The two versions of the program were not assigned randomly, but rather in consultation with primary care leadership and with a goal of balancing the type of practice assigned (e.g., community health center, hospital-based clinic) between the two versions of the program, while also achieving buy-in from the practice 
leaders. Of the 18 practices, 8 were assigned to version one (PHC practices) and 10 to version two (non-PHC practices).

\section{Outcomes}

Outcomes were assessed using data from the participants' clinical record. For LDL analyses, participants were considered to have met their goal if they were prescribed a high-dose HMG coreducatase inhibitor (statin) in the preceding 1 year period or had an LDL level under $100 \mathrm{mg} / \mathrm{dl}$. For BP analyses (see eFigure 1 for flowchart), goals were concordant with JNC 8 Guidelines. ${ }^{23}$ Participants who were under 60 years of age met their goal if their systolic blood pressure was $<140 \mathrm{mmHg}$ and their diastolic blood pressure was $<90 \mathrm{mmHg}$ or they were prescribed three or more anti-hypertensive agents. Those aged 60 and older met their goal as long as they had a diastolic blood pressure < $70 \mathrm{mmHg}$ regardless of other factors, owing to the risk associated with further BP lowering in this group. ${ }^{24}$ Additionally, if their diastolic blood pressure was $\geq 70$ but $<90 \mathrm{mmHg}$ and their systolic blood pressure was $<150 \mathrm{mmHg}$, or if they were prescribed three or more anti-hypertensive agents from different classes, they were considered to be at goal. ${ }^{10}$

\section{Statistical Analysis}

We first performed descriptive statistics. A prior manuscript reported the overall outcomes for this intervention, but did not examine changes in disparities. ${ }^{10}$ Our goal for this project was to determine changes in the proportion meeting LDL and BP goals, by race/ethnicity, before (July 1, 2014) and after (December 31,2014 ) the intervention periods. To do this, we used a difference-in-difference approach. This involved comparing clinics with the two versions of the population health management program both before and after implementation and examining whether the disparity between racial/ethnic minority groups and non-Hispanic white patients decreased. The primary independent variable was the version of the population health management program the practice used (PHC vs. nonPHC) and we adjusted for age, gender, health insurance, primary language, patient-physician continuity, ${ }^{25}$ and whether care was received in a health center in multivariable models To determine whether there was differential improvement in disparities for those patients in a PHC clinic, we also tested the three-way interaction (PHC vs. non-PHC, before program implementation vs. after, and race/ethnicity) in the models. All analyses were conducted in SAS version 9.4 (SAS Institute, Cary, NC). We used logistic regression with generalized estimating equations to account for repeated measurements within patients. A two-sided $P$ value of 0.05 or less was considered statistically significant.

\section{RESULTS}

In the LDL analysis, 12,555 participants met the criteria for cardiovascular disease, had self-reported race/ethnicity data available, and were in their practice at the start and end of the study period. Of this cohort, $85.5 \%$ identified as non-Hispanic white, $4.8 \%$ as non-Hispanic black, and $4.7 \%$ as Hispanic. Following similar criteria, 41,183 participants were included in the BP analyses, of whom $79.7 \%$ identified as non-Hispanic white, $7.5 \%$ as non-Hispanic black, and $6.6 \%$ as Hispanic. Compared with non-Hispanic white patients, both non-Hispanic black and Hispanic patients were more likely to have Medicaid insurance and have a primary language other than English. Approximately one third of participants in both the LDL and $\mathrm{BP}$ analysis received care in a $\mathrm{PHC}$ practice (Table 1).

\section{LDL Control}

At baseline, there was a disparity in the proportion of patients at LDL goal by race/ethnicity; $70.3 \%$ of non-Hispanic white, $64.2 \%$ of non-Hispanic black, and $68.5 \%$ of Hispanic patients $(p=0.007)$ were at goal. Over the course of the study period, LDL control improved in all groups (Fig. 1 and Table 2, absolute changes). When examining changes, there are several relevant comparisons. First, we examined LDL control comparing after with before the intervention, stratified by race/ ethnicity and whether the practice was a PHC or non-PHC practice. Compared with pre-intervention, non-Hispanic white patients in both the PHC and non-PHC versions of the program made significant improvements in LDL control postintervention, (adjusted odds ratio [aOR] 1.60 (95\% confidence interval [CI] 1.48-1.74 and aOR 1.34, 95\% CI 1.27-1.40, respectively). The change among non-Hispanic black (aOR $1.36,95 \%$ CI $0.99-1.85$ ) or Hispanic patients (aOR 1.18, 95\% CI 0.98-1.43) was similar in magnitude but not statistically significant, possibly because of the smaller sample sizes (Table 2, relative changes, PHC or non-PHC).

Next, we examined whether, within racial/ethnic groups, changes in LDL control were greater in PHC, compared with non-PHC, practices (Table 2, relative changes, PHC vs. non$\mathrm{PHC})$. Compared with patients from non-PHC practices, nonHispanic white patients in the PHC practices were more likely to be at goal for LDL control, (aOR 1.20, 95\% CI 1.09-1.32) after intervention. Improvement similar in magnitude, though not statistically significant, was seen among non-Hispanic black (aOR 1.15, 95\% CI 0.80-1.65) and Hispanic patients (aOR 1.29, 95\% CI 0.66-2.53) in PHC compared with nonPHC practices.

Finally, we compared, across racial/ethnic groups, whether the change for racial/ethnic minority groups was different from that of non-Hispanic white patients (Table 2, comparing changes across racial/ethnic groups). We found no evidence of differential improvement in meeting LDL goals, as would be needed to reduce disparities, in patients from PHC, compared with non-PHC, practices ( $\mathrm{p}$ for 3-way interaction = 0.73 ). Both non-Hispanic black patients (aOR $0.98,95 \% \mathrm{CI}$ 0.67-1.43) and Hispanic patients (aOR 1.05, 95\%CI 0.542.06) had odds of improvement similar to non-Hispanic white patients in $\mathrm{PHC}$ vs. non-PHC practices. 
Table 1 Demographic Characteristics of Participants

\begin{tabular}{|c|c|c|c|c|c|c|}
\hline & \multicolumn{2}{|c|}{ Non-Hispanic black } & \multicolumn{2}{|l|}{ Hispanic } & \multicolumn{2}{|c|}{ Non-Hispanic white } \\
\hline & NON-PHC & PHC & NON-PHC & PHC & NON-PHC & PHC \\
\hline & \multicolumn{6}{|c|}{ LDL population } \\
\hline $\mathrm{N}$ & 416 & 191 & 479 & 112 & 7230 & 3507 \\
\hline Age (SD) & $68.3(12.1)$ & $63.8(12.5)$ & $65.2(13.3)$ & $63.8(13.8)$ & $72.6(11.9)$ & $70.7(12.0)$ \\
\hline Female $(\%)$ & 53.8 & 51.8 & 48.9 & 51.8 & 35.7 & 40.1 \\
\hline Non-English primary language $(\%)$ & 11.5 & 8.9 & 73.5 & 58.9 & 3.2 & 2.3 \\
\hline \multicolumn{7}{|l|}{ Insurance $(\%)$} \\
\hline Private & 29.1 & 46.6 & 33.2 & 31.3 & 32.1 & 35.9 \\
\hline Medicaid & 14.7 & 14.7 & 23.2 & 17.9 & 4.8 & 5.6 \\
\hline Medicare & 54.8 & 38.2 & 42.0 & 49.1 & 62.7 & 58.1 \\
\hline Uninsured & 1.4 & 0.5 & 1.7 & 1.8 & 0.5 & 0.4 \\
\hline Receive care in health center $(\%)$ & 11.1 & 19.4 & 61.2 & 58.0 & 15.6 & 37.4 \\
\hline Patient-physician connected & 86.3 & 92.1 & 92.5 & 89.3 & 92.9 & 95.6 \\
\hline \multicolumn{7}{|l|}{ Comorbidity $(\%)$} \\
\hline Diabetes mellitus & 50.5 & 48.2 & 51.2 & 40.2 & 27.3 & 27.4 \\
\hline Obesity & 48.3 & 51.8 & 48.9 & 50.9 & 39.3 & 40.8 \\
\hline Congestive heart failure & 20.9 & 21.5 & 21.7 & 18.8 & 17.9 & 18.0 \\
\hline Charlson score (SD) & $5.4(2.9)$ & $4.7(2.5)$ & $5.3(2.9)$ & $5.2(2.8)$ & $5.3(2.9)$ & $5.1(2.9)$ \\
\hline \multicolumn{7}{|l|}{ BP population } \\
\hline $\mathrm{N}^{1}$ & 2018 & 1079 & 2190 & 544 & 21,001 & 11,828 \\
\hline Age (SD) & $61.5(13.4)$ & $58.4(12.4)$ & $58.1(13.8)$ & $55.9(13.3)$ & $67.2(13.4)$ & $64.6(13.0)$ \\
\hline Female $(\%)$ & 56.5 & 61.5 & 55.7 & 54.8 & 47.0 & 53.7 \\
\hline Non-English primary language $(\%)$ & 13.8 & 8.1 & 71.1 & 51.5 & 3.2 & 1.9 \\
\hline \multicolumn{7}{|l|}{ Insurance $(\%)$} \\
\hline Private & 48.7 & 59.5 & 46.0 & 46.3 & 46.3 & 52.7 \\
\hline Medicaid & 17.2 & 16.6 & 27.9 & 26.5 & 5.6 & 6.0 \\
\hline Medicare & 31.9 & 21.8 & 24.1 & 25.7 & 47.4 & 40.6 \\
\hline Uninsured & 2.2 & 2.1 & 2.0 & 1.5 & 0.7 & 0.7 \\
\hline Receive care in health center $(\%)$ & 15.7 & 20.7 & 68.2 & 60.3 & 14.4 & 30.7 \\
\hline Patient-physician continuity* & 89.1 & 91.8 & 93.3 & 90.1 & 93.3 & 95.3 \\
\hline \multicolumn{7}{|l|}{ Comorbidity $(\%)$} \\
\hline Diabetes mellitus & 34.7 & 34.1 & 38.5 & 35.1 & 19.8 & 19.1 \\
\hline Obesity & 51.6 & 54.1 & 54.2 & 59.0 & 43.4 & 46.9 \\
\hline Congestive heart failure & 6.5 & 6.0 & 5.8 & 3.1 & 7.1 & 6.4 \\
\hline Charlson score (SD) & $3.2(2.4)$ & $2.9(2.2)$ & $3.3(2.4)$ & $3.2(2.3)$ & $3.7(2.6)$ & $3.4(2.5)$ \\
\hline
\end{tabular}

PHC population health coordinator

*Patient-physician continuity represents the proportion of patients who can be attributed to a specific continuity physician as opposed to being seen at the practice without being clearly empaneled with a particular, based on a validated algorithm ${ }^{25}$

\section{BP Control}

Our analyses for BP control followed the same order as reported for LDL analyses. At baseline, a small disparity was present in the proportion of patients at BP goal by race/ ethnicity; $76.4 \%$ of non-Hispanic white, $73.4 \%$ of nonHispanic black, and $74.8 \%$ of Hispanic patients $(p=0.0003)$. Over the course of the study period, BP control improved in non-Hispanic white patients in both the PHC (aOR 1.23, 95\% CI 1.16-1.31) and non-PHC (aOR 1.09, 95\% CI 1.04-1.14) practices (Fig. 2, Table 2, relative changes). When compared with non-PHC practices, non-Hispanic white patients from PHC practices demonstrated improved BP control (aOR $1.13,95 \%$ CI 1.05-1.22) after intervention. The improvement in $\mathrm{PHC}$ practices compared with non-PHC practices for nonHispanic black patients (aOR 1.17, 95\% CI 0.94-1.45) was similar in magnitude, but not statistically significant (Table 2). The changes for Hispanic patients were qualitatively different (aOR 0.90, 95\% CI 0.59-1.36).

We found no evidence of differential improvement in meeting BP goals, as would be needed to reduce disparities, in patients from $\mathrm{PHC}$ compared with non-PHC practices ( $\mathrm{p}$ for 3way interaction $=0.69$ ). Neither non-Hispanic black patients (aOR 1.05, 95\% CI 0.83-1.31) nor Hispanic patients (aOR
$0.82,95 \%$ CI $0.53-1.25$ ) had significantly different odds of improvement in PHC, vs. non-PHC practices, compared with non-Hispanic white patients.

\section{DISCUSSION}

In this study, the overall implementation of a population health management program showed statistically significant improvement in LDL and BP control. ${ }^{10}$ For non-Hispanic white patients, improvements were greater for patients in practices with a central population health coordinator. Changes were generally similar in magnitude, but not statistically significant, for non-Hispanic black and Hispanic patients, except for BP control in Hispanic patients. Interaction testing revealed that the PHCs did not help 'close the gap' in BP or LDL control for non-Hispanic black or Hispanic patients.

There is a growing body of literature on the overall effectiveness of population health management programs at improving healthcare screening and chronic disease management. ${ }^{8-10}$ Less is known about the impact these programs have on health disparities. Two population health management programs have demonstrated narrowing of health disparities in chronic disease care, ${ }^{13,14}$ but many others have not. ${ }^{15-19}$ 


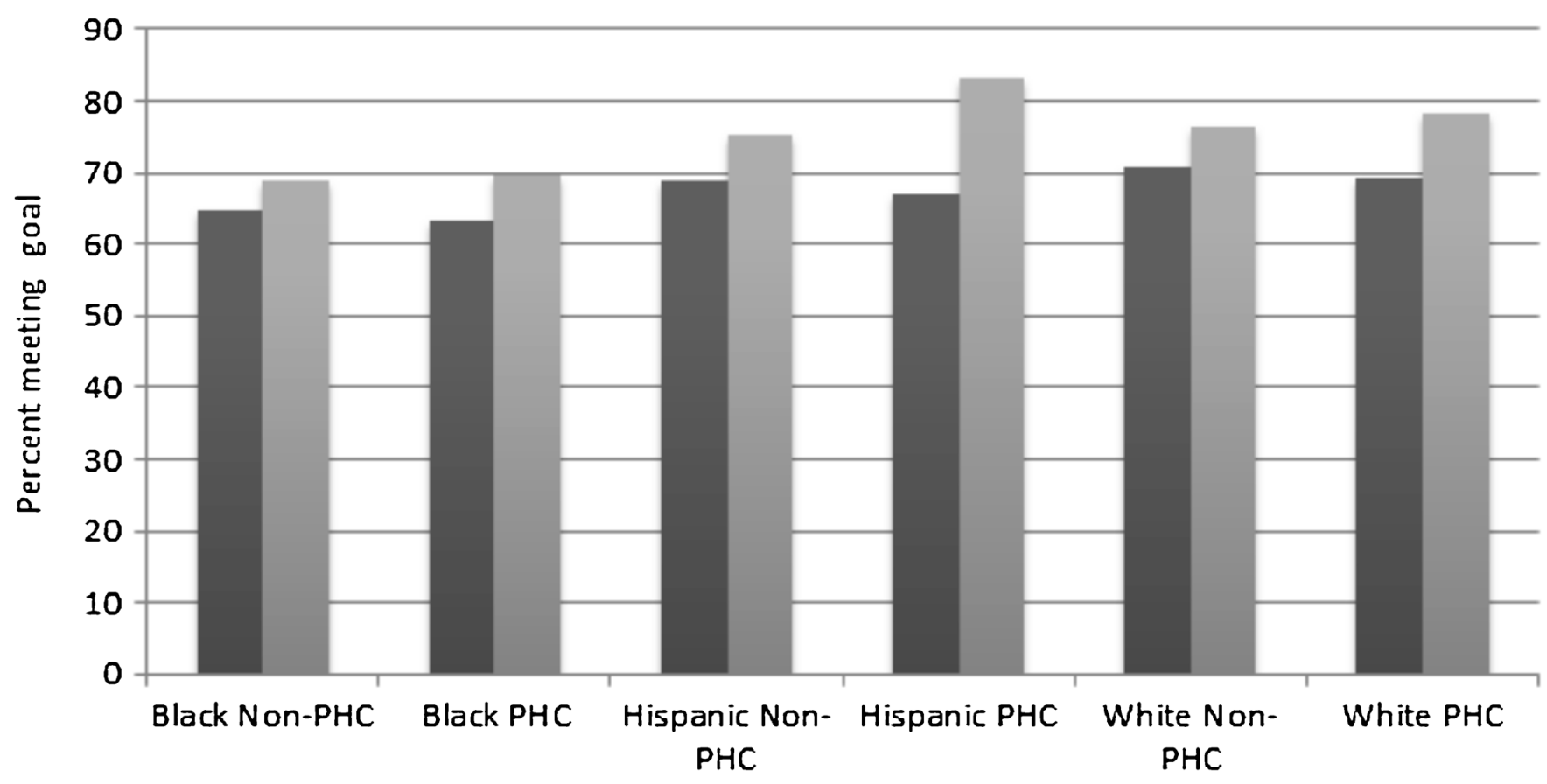

$7 / 1 / 14=12 / 31 / 14$

Figure 1 Percentage of participants meeting LDL cholesterol goal by time, intervention group, and race/ethnicity

Our results are consistent with the latter. The Veterans Health Administration and a large urban health system implemented population health management programs using reminders and clinical decision tools. Both programs improved overall quality in several chronic disease measures including blood pressure and cholesterol control, but racial and ethnic disparities

Table 2 Absolute and Relative Changes in Meeting Goal, by Time, Group, and Race/Ethnicity

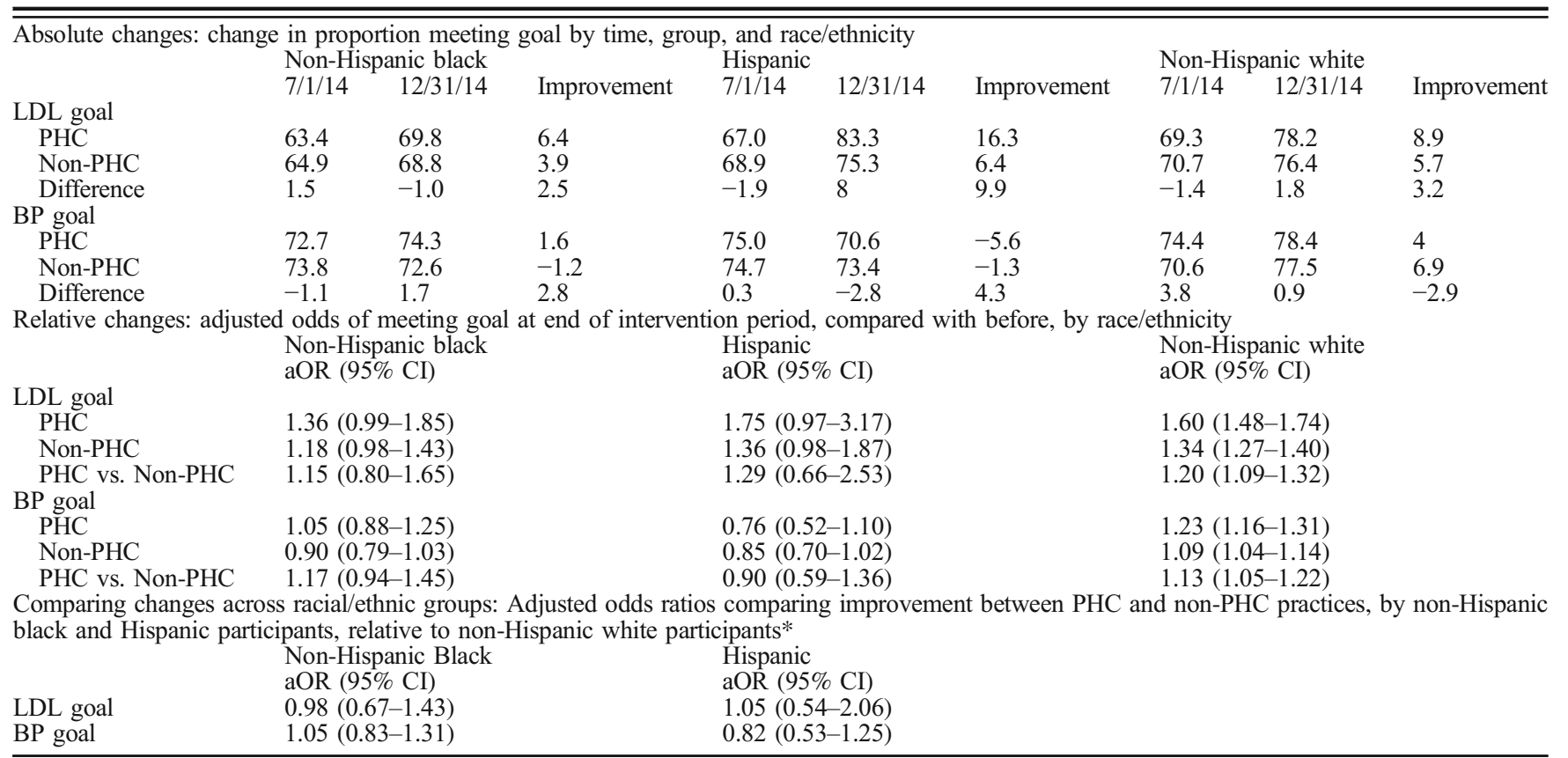

PHC population health coordinator, aOR adjusted odds ratio, 95\% CI, 95\% confidence interval. Adjusted for age, gender, primary language, health insurance, connection to clinic, and receipt of care in a health center

*This comparison is analogous to a three-way interaction with terms for time (before vs. after intervention), type of intervention (PHC vs. non-PHC practice), and racelethnicity (non-Hispanic black, Hispanic, non-Hispanic white (the reference category)]. The odds ratio quantifies how the odds of being at goal for non-Hispanic black or Hispanic patients in PHC practices after the intervention relates the odds of being at goal for non-Hispanic white patients in PHC practices after the intervention. For disparities to improve, the odds would need to be significantly greater for racial/ethnical minority patients (odds ration > 1.0) because at baseline non-Hispanic white patients were more likely to be at goal 


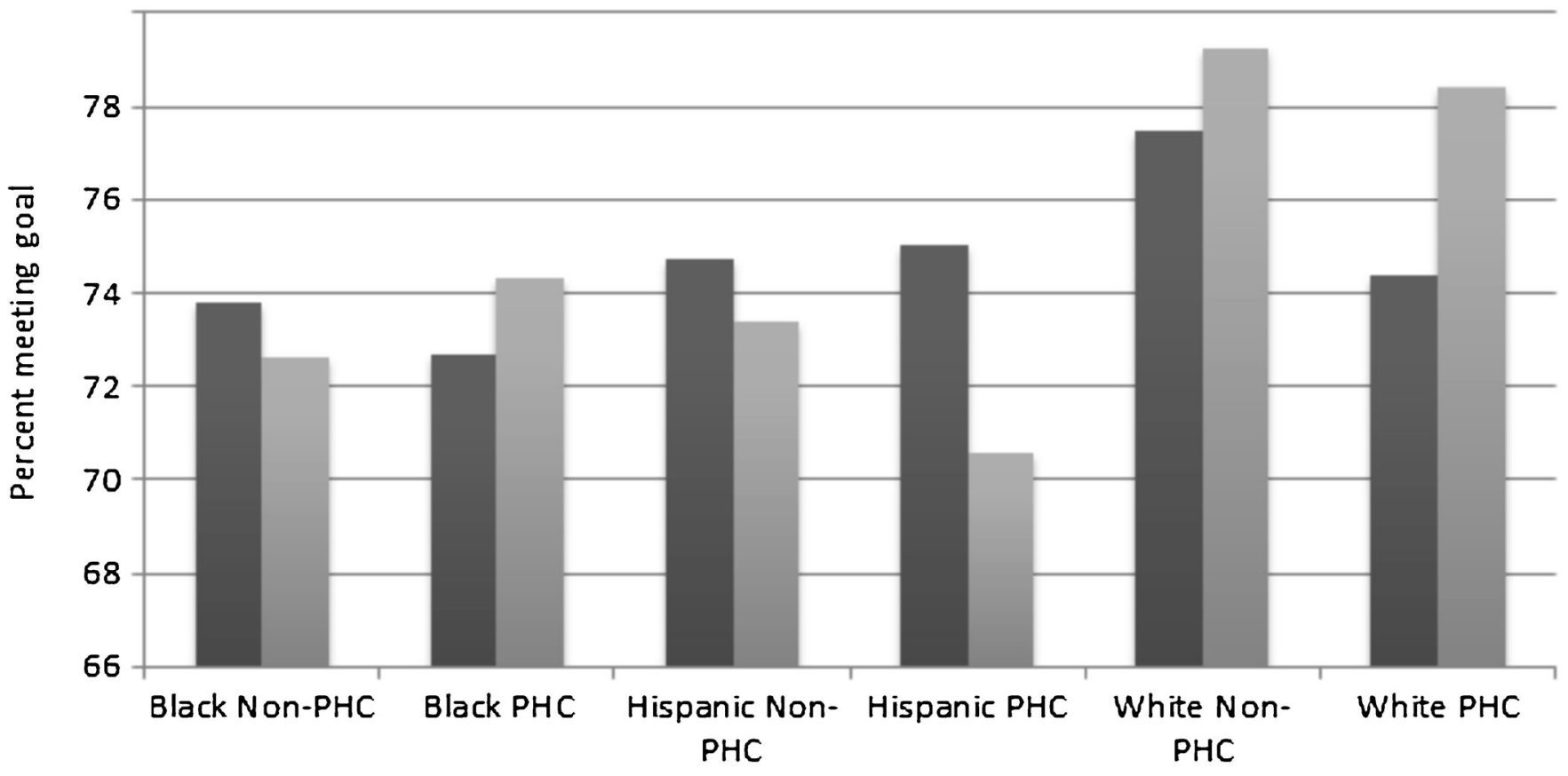

$7 / 1 / 14 \square 12 / 31 / 14$

Figure 2 Percentage of participants meeting blood pressure goal by time, intervention group, and race/ethnicity

persisted. ${ }^{15,16}$ However, these prior studies did not evaluate the addition of a dedicated population health management workforce. The current study presents new evidence that this additional feature - while improving overall quality — may not be sufficient to reduce racial/ethnic disparities in cardiovascular risk factor control. In contrast to chronic disease management, more studies of population management interventions for cancer screening have shown narrowing of disparities. ${ }^{26,27}$ One explanation for this discrepancy may be that disease screening is often a single discrete task, such as obtaining a mammogram, whereas achieving control of a chronic disease typically requires multiple appointments, medication changes, and patient counseling.

The difficulty in simultaneously improving quality and reducing disparities in chronic disease management is an important finding with implications for future research on improving healthcare delivery. While quality improvement efforts are widespread, equity improvement efforts are less so, and even effective quality improvement interventions may not 'lift all boats' with regard to achieving equity goals. Interestingly, programs designed to address health disparities have been found to be effective in improving blood pressure and lipid control in both racial/ethnic minorities and the overall population. ${ }^{28,29}$ We believe that including specific and explicit strategies aimed at narrowing inequality should be a fundamental part of the design of population health programs. These strategies may include the addition of tools to overcome socioeconomic barriers to care, as racial/ethnic minority patients may disproportionately face these barriers, ${ }^{30}$ and improve access to care, patient engagement, and patient-provider communication. Adding community-based participation, in which those individuals the program will serve are included in the design, implementation, and evaluation of the program, may be a fruitful way to improve these programs. Other directions for future research include examining the role differences age and gender may play in population health management efforts and using qualitative methods to investigate the experiences of different demographic groups within the program.

This study has several important limitations. The 6-month study period, while adequate to determine the effect of the intervention, is relatively short for assessing outcomes in chronic conditions such as hypertension or high LDL cholesterol. Some patients may have come into control over a longer period, though we do not expect there would be differences between PHC and non-PHC practices in the proportion of the patients who do so. Because PHCs were expanded to all practices at the end of the 6-month study period, we were unable to analyze a longer timeframe. This study focused on dichotomous treatment goals, but some patients, particularly those with very elevated LDL and BP, may receive clinical benefit even if they remain above goal, which would not be captured in our analysis. Alternative quality indicators, which capture the clinical value of improvement even for patients who do not meet a particular threshold, may be one way to better capture the clinical benefit of population health management programs. ${ }^{31}$ As a single healthcare delivery network, the results may not be generalizable to other settings, particularly with different racial/ethnic composition. However, the network does include a diversity of practice types including 
community health centers, private practice, as well as teaching and non-teaching practices. It is also important to note that the magnitude of racial/ethnic disparities in our population is less than the national average. ${ }^{4-6}$ Thus, the effect of similar programs may be different in other settings. Due to limitations of the data source, we were not able to evaluate potential mechanisms of disparities in LDL and BP control, such as reduced medication adherence, food insecurity, or other barriers. Who benefits from LDL control as well as what level to target is an area of ongoing controversy, and changes have occurred since the study period. ${ }^{32,33}$ While the same target was applied throughout this study, how our results would apply to different LDL control strategies that may be developed is unclear. Finally, the relatively lower number of non-Hispanic black and Hispanic participants may have reduced power to detect differences.

These limitations are balanced by several strengths. This study evaluated the real-world implementation of a population management program consisting of a population health management information technology system with or without a dedicated population health coordinator in a large and diverse primary care network, suggesting similar programs may be feasible in routine care. Furthermore, the linking of population-level improvements in quality metrics to pay-for-performance contracts suggests a possible financial mechanism to implement programs that improve equity of care.

This study found that a multi-faceted population health management intervention, including both information technology and workforce components, did show overall improvements, but was not sufficient to reduce disparities in chronic disease measures. To improve both healthcare quality and equity, we believe future population health management interventions should explicitly focus on decreasing disparities in care.

Acknowledgements: Funding for this program, including the IT tool and personnel, was provided by the Massachusetts General Physicians Organization and Partners HealthCare. These funders were involved in creating and implementing the program, but the authors were responsible for design and conduct of the study; collection, management, analysis, and interpretation of the data; and preparation, review, and submission of the manuscript without funder approval. Seth A. Berkowitz's role in the research reported in this publication was supported by the National Institute of Diabetes and Digestive and Kidney Diseases of the National Institutes of Health under Award No. K23DK109200. The content is solely the responsibility of the authors and does not necessarily represent the official views of the National Institutes of Health.

Corresponding Author: Seth A. Berkowitz, MD MPH; Division of General Internal Medicine Massachusetts General Hospital, Boston, MA, USA (e-mail: saberkowitz@partners.org).

\section{Compliance with Ethical Standards:}

Conflict of Interest: Massachusetts General Hospital entered into a royalty arrangement on June 27,2013 , to commercialize the population management system with SRG Technology, a for-profit company. Dr. Atlas is a beneficiary of this royalty arrangement but has not received any payments to date. Dr. Atlas has received payments as a consultant for the company. All other authors declare that they have no disclosures.
Dr. Berkowitz had full access to all of the data in the study and takes responsibility for the integrity of the data and accuracy of the data analysis.

Prior Presentation: A version of the study was presented as an Oral Abstract at the Society for General Internal Medicine Meeting in Washington, DC, on April 21, 2017.

\section{REFERENCES}

1. National Center for Health Statistics. Health, United States, 2015: With special feature on racial and ethnic health disparities. Hyattsville, MD. 2016. https://www.cdc.gov/nchs/data/hus/hus15.pdf. Accessed 3 Jan 2018.

2. Fang J, Yang $\mathbf{Q}$, Hong $\mathbf{Y}$, Loustalot F. Status of cardiovascular health among adult Americans in the 50 states and the District of Columbia, 2009. J Am Heart Assoc. 2012;1(6):e005371-e005371.

3. Golf DC, Lloyd-Jones DM, Bennett G, Coady S, D'Agostino RB, Gibbons R, ... Wilson PWF. 2013 ACC/AHA Guideline on the Assessment of Cardiovascular Risk: a Report of the American College of Cardiology/American Heart Association Task Force on Practice Guidelines. Circulation. 2014;129(25 suppl 2):S49-S73.

4. Centers for Disease Control and Prevention (CDC). Health disparities and inequalities report-United States. MMWR Morb Mortal Wkly Rep. 2013;62 Suppl 3:157-60.

5. Mozaffarian D, Benjamin EJ, Go AS, Arnett DK, Blaha MJ, Cushman M, ... Turner MB. Heart Disease and Stroke Statistics-2016 Update: a Report From the American Heart Association. Circulation. 2016;133(4):e38-e360.

6. Yoon SS, Burt V, Louis T, Carroll MD. Hypertension among adults in the United States, 2009-2010. NCHS Data Brief. 2012;(107):1-8.

7. A Public Health Action Plan to Prevent Heart Disease and Stroke. The US Department of Health and Human Services, Center for Disease Control. http://www.cdc.gov/dhdsp/action_plan/pdfs/action_plan_full.pdf, Access date April 2017.

8. Jamal A, McKenzie K, Clark M. The impact of health information technology on the quality of medical and health care: a systematic review. HIM J. 2009;38(3):26-37.

9. Chaudhry B, Wang J, Wu S, Maglione M, Mojica W, Roth E, ... Shekelle PG. Systematic review: impact of health information technology on quality, efficiency, and costs of medical care. Ann Intern Med. 2006; 144(10):742-752.

10. Ashburner JM, Horn DM, O'Keefe SM, Zai AH, Chang Y, Wagle NW, Atlas SJ. Chronic disease outcomes from primary care population health program implementation. Am J Manag Care. 2017; In press.

11. Trivedi AN, Nsa W, Hausmann LRM, Lee JS, Ma A, Bratzler DW, ... Fine MJ. Quality and equity of care in US hospitals. N Engl J Med. 2014;371(24):2298-2308.

12. Sehgal AR. Impact of quality improvement efforts on race and sex disparities in hemodialysis. JAMA. 2003;289(8):996-1000.

13. Jackson GL, Oddone EZ, Olsen MK, Powers BJ, Grubber JM, McCant F, Bosworth HB. Racial differences in the effect of a telephone-delivered hypertension disease management program. J Gen Intern Med. 2012;27(12):1682-1689.

14. Bosworth HB, Olsen MK, Grubber JM, Powers BJ, Oddone EZ. Racial differences in two self-management hypertension interventions. Am J Med. 2011;124(5):468.e1-8.

15. Trivedi AN, Grebla RC, Wright SM, Washington DL. Despite improved quality of care in the Veterans Affairs health system, racial disparity persists for important clinical outcomes. Health Aff (Proj Hope). 2011;30(4):707-715.

16. Jean-Jacques M, Persell SD, Thompson JA, Hasnain-Wynia R, Baker Dw. Changes in disparities following the implementation of a health information technology-supported quality improvement initiative. J Gen Intern Med. 2012;27(1):71-77.

17. Sequist TD, Adams A, Zhang F, Ross-Degnan D, Ayanian JZ. Effect of quality improvement on racial disparities in diabetes care. Arch Intern Med. 2006;166(6):675-681.

18. Miranda J, Duan N, Sherbourne C, Schoenbaum M, Lagomasino I, Jackson-Triche M, Wells KB. Improving care for minorities: can quality improvement interventions improve care and outcomes for depressed minorities? Results of a randomized, controlled trial. Health Serv Res. 2003;38(2):613-630.

19. Trief PM, Izquierdo R, Eimicke JP, Teresi JA, Goland R, Palmas W, ... Weinstock RS. Adherence to diabetes self care for white, African- 
American and Hispanic American telemedicine participants: 5 year results from the IDEATel project. Ethn Health. 2013;18(1):83-96.

20. Howe GR, Chiarelli AM. Methodological issues in cohort studies. II: Power calculations. Int J Epidemiol. 1988;17(2):464-468.

21. Leong A, Berkowitz SA, Triant VA, Porneala B, He W, Atlas SJ, ... Meigs JB. Hypoglycemia in diabetes mellitus as a coronary artery disease risk factor in patients at elevated vascular risk. J Clin Endocrinol Metab. 2016;101(2):659-668.

22. Berkowitz SA, Hulberg AC, Hong C, Stowell BJ, Tirozzi KJ, Traore CY, Atlas SJ. Addressing basic resource needs to improve primary care quality: a community collaboration programme. BMJ Qual Saf. 2016;25(3):164-172.

23. Friedmann H, Lubart R, Laulicht I, Rochkind S. A possible explanation of laser-induced stimulation and damage of cell cultures. J Photochem Photobiol B. 1991;11(1):87-91.

24. McEvoy JW, Chen Y, Rawlings A, Hoogeveen RC, Ballantyne CM, Blumenthal RS, ... Selvin E. Diastolic blood pressure, subclinical myocardial damage, and cardiac events: implications for blood pressure control. J Am Coll Cardiol. 2016;68(16):1713-1722. https://doi.org/10. 1016/j.jacc.2016.07.754.

25. Atlas SJ, Grant RW, Ferris TG, Chang Y, Barry MJ. Patient-physician connectedness and quality of primary care. Ann Intern Med. 2009; 150(5):325-335.

26. Berkowitz SA, Percac-Lima S, Ashburner JM, Chang Y, Zai AH, He W, ... Atlas SJ. Building equity improvement into quality improvement: reducing socioeconomic disparities in colorectal cancer screening as part of population health management. J Gen Intern Med, 2015;30(7):942-949.
27. Lasser KE, Murillo J, Lisboa S, Casimir AN, Valley-Shah L, Emmons KM, ... Ayanian JZ. Colorectal cancer screening among ethnically diverse, low-income patients: a randomized controlled trial. Arch Intern Med. 2011;171(10):906-912.

28. Bartolome RE, Chen A, Handler J, Platt ST, Gould B. Population care management and team-based approach to reduce racial disparities among African Americans/Blacks with hypertension. Permanente J. 2016;20(1):53-59.

29. Mahotière T, Ocepek-Welikson $\mathbf{K}$, Daley MB, Byssainthe JP. A program to reduce the disparity in the rate of biennial lipid profiles between African-American and white Medicare beneficiaries with diabetes mellitus in New York City. J Community Health. 2006;31(4):263-288.

30. Avoni A, Prati C, Montanari MC. [AIDS: notes on the prevention of HTLV-III virus infection in the dental field]. Minerva Stomatol. 1986;35(11):1037-1041.

31. Baxi S, Lakin J, Lyles CR, Berkowitz S, Horton C, Sarkar U. Points for improvement: performance measurement for glycemic control in diabetes patients in a safety-net population. Jt Comm J Qual Patient Saf. 2013;39(3): 109-113.

32. Beck ML, Freihaut B, Henry R, Pierce S, Bayer WL. A serum haemagglutinating property dependent upon polycarboxyl groups. $\mathrm{Br} \mathrm{J}$ Haematol. 1975;29(1): 149-156.

33. US Preventive Services Task Force, Bibbins-Domimgo K, Grossman DC, Curry SJ, Davidson KW, Epling JW, ... Pignone MP. Statin use for the primary prevention of cardiovascular disease in adults: US Preventive Services Task Force Recommendation Statement. JAMA. 2016;316(19):1997-2007. 